\title{
TRAYECTORIAS DE ABOGADOS PROFESIONALES Y ACADÉMICOS: EL CASO DE UNA UNIVERSIDAD PÚBLICA MEXICANA Y SU ENTORNO
}

\section{Introducción}

En los últimos años las instituciones de educación superior en el mundo y en nuestro país se han visto inmersas en una serie de cuestionamientos sobre su funcionamiento, sobre todo referente a su respuesta ante las demandas de diferentes instancias, como el gobierno, la industria y los diferentes grupos sociales de su entorno. Tales demandas están influyendo en las formas de hacer investigación, docencia y de abordar la formación de profesionales, tareas que, con el paso del tiempo, se han hecho cada vez más complejas de realizar dentro de dichas instituciones (Clark, 2004). Al respecto, diferentes investigadores han señalado la diversidad y relevancia de la profesión académica y se ha incrementado el número de estudios relacionados con ella (García Salord, Landesmann y Gil Antón, 1993; Gil Antón et al., 1994), aunque todavía quedan muchos aspectos por explorar y muchos temas en los cuales se requiere profundizar el análisis.

Un tema que hasta este momento ha sido relativamente poco estudiado de una manera directa, en el caso de México, es el relacionado con la cultura de la profesión académica, uno de cuyos aspectos centrales es la trayectoria profesional de sus integrantes y los procesos que allí subyacen. En algunos estudios y reflexiones sobre la cultura académica se han abordado aspectos como las tareas básicas del trabajo académico (Garritz Ruiz, 1997), la participación de los académicos en la toma de decisiones (Tapia Uribe, 2000; Galaz Fontes y Viloria Hernández, 2004; Gil Antón, 2000), los criterios para avanzar dentro de la carrera académica (Villa Lever, 2001) y otros. No obstante, no hay estudios empíricos que, de manera integral, aborden la caracterización de las trayectorias de los docentes como elemento de la cultura académica. 
El concepto de "cultura académica" se importó de la teoría organizacional, la cual a su vez lo tomó de la antropología, y su importancia reside en el hecho de que contribuye a una mejor comprensión del comportamiento individual y organizacional, así como de las actitudes de los integrantes de una organización hacia la misma (Grediaga Kuri, 2001; Padilla González, 2007; Schein, 1989; Tierney, 1997). Por otro lado, el concepto de "profesión académica" ha sido utilizado por Clark (1987) para describir la vida académica en instituciones de educación superior estadounidenses, en las cuales identificó valores como la honestidad intelectual, la libertad de cátedra, la noción de comunidad de expertos y jerarquías de prestigio en el marco de la profesión. Becher y Trowler (2001), por su cuenta, han utilizado los conceptos de "tribus" y "territorios" como una analogía en su análisis de las disciplinas. Por último, Austin (1989) ha trabajado la noción de "cultura académica" en términos de creencias, compromisos y conductas, así como de algunos valores que parecen unir a los académicos, particularmente los asociados a una misma profesión y/o disciplina.

Por otro lado, al hacer referencia al concepto de "cultura" también se alude a la noción de "cultura organizacional", la cual se concibe como el conjunto de normas, creencias y valores que crean, comparten y practican los individuos de una organización. Estos procesos dan identidad y sentido a sus comportamientos, los cuales se manifiestan en elementos simbólicos y materiales, como mitos, rituales, lenguaje especializado e instrumentos utilizados, los cuales son compartidos por los miembros de la organización (Robbins, 2004; Schein, 2004; Tierney, 1993).

En México, la investigación sobre las trayectorias académicas cobra relevancia por los cambios vividos en las universidades públicas con el constante crecimiento, tanto de su matrícula como del mercado académico asociado (Gil Antón et al., 1994; Landesmann, 2001). Las formas de aproximación teórica y metodológica sobre las trayectorias académicas son muy variadas, lo que ha permitido el desarrollo de aproximaciones tanto cuantitativas como cualitativas, las cuales han contribuido a dar cuenta de tan complejo proceso. 
Entre las investigaciones sobre las trayectorias profesionales de los académicos en México se encuentra la de Landesmann (2001), quien aborda la comprensión del oficio desde la pertenencia institucional, el tipo de contrato, la afiliación disciplinaria y la trayectoria desde su reconstrucción histórica, al estudiar tres generaciones de académicos y la diversificación de los roles que desarrollan como gestores, investigadores y profesores. García Salord (2001), por su parte, explica la diversidad y heterogeneidad de las trayectorias académicas mediante la descripción de los procesos generacionales en los que se construye la desigualdad, la diferencia y la discrepancia al interior de los núcleos de grupos dentro de la institución donde se desarrollan las diferentes trayectorias, en los cuales predomina la verticalidad organizacional y una debilidad en el plano horizontal de los patrones de constitución y reproducción de la carrera académica (García Salord, 2001).

También se ha estudiado el papel de las políticas públicas en México y su influencia en el desarrollo de la profesión académica. Las variables para explicar la decisión de concentrarse en la vida académica por parte de los profesionales se relacionan con el acceso a los recursos para hacer investigación, el grado académico, el tipo de socialización ocurrido al ingresar al trabajo y la remuneración vinculada a la estabilidad en el empleo y tipo de actividad (Grediaga Kuri, 2001).

Dentro del contexto institucional, la reconstrucción de las trayectorias académicas ha sido abordada desde el proceso de socialización organizacional, en el cual se destaca la incorporación al mercado, la obtención de la definitividad y la promoción. Aquí los académicos valoran la socialización recibida por parte de otros pares, la posibilidad de formación y la seguridad en el empleo (Villa Lever, 2001).

Otro aspecto asociado con las trayectorias académicas es la organización del trabajo en la disciplina, la institución y el contexto organizacional. Estas son condiciones clave que ejercen importante influencia en las creencias y valores sobre las formas de organizar sus actividades y, en lo particular, incorporarse a las actividades de investigación (Chavoya, 2001). 
De acuerdo con Light (1990), la "trayectoria" se concibe como una serie de etapas sucesivas de puestos de trabajo o actividades laborales que se ordenan, idealmente, en forma ascendente y jerárquica, y que experimentan las personas a lo largo de su historia personal y laboral. En muchos casos, las trayectorias profesionales implican una formación especializada en un área del conocimiento que facilita a la persona escalar a un puesto o actividad de mayor jerarquía. Así, las trayectorias profesionales implican aspectos de tiempo y desarrollo. El tiempo se refiere a la sucesión de acontecimientos (ingreso a la carrera, incorporación al trabajo) que determinan periodos más o menos identificables. El desarrollo, por otro lado, se refiere a cambios progresivos, puede ser lineal o no serlo, y pasa generalmente por etapas (Galaz Fontes, 2003; García Salord, 2001; Padilla González, 2007; y Vargas Leyva, 2000).

Además de considerar la dimensión personal de toda trayectoria profesional, es importante reconocer que ésta siempre se vive bajo un contexto social e histórico determinado. Así, dicha trayectoria se delimita por la clase social y origen socioeconómico del involucrado, por la institución en la que realiza sus estudios y, de una manera más amplia, por la situación económica y política de su entorno, la cual establece oportunidades de trabajo, de ascenso y, algo fundamental, una cultural general con un discurso y mecanismos concretos de acuerdo con los cuales los individuos avanzan en sus carreras. Como es característico de muchos ámbitos del quehacer humano, el discurso y los mecanismos implicados en las trayectorias profesionales no siempre concuerdan.

Desde dicha perspectiva, el estudio de las trayectorias profesionales y académicas permite un acercamiento para comprender y explicar el proceso mediante el cual los abogados exitosos ocupan diferentes puestos a lo largo del tiempo, adquieren prestigio y poder. Estudiando los casos de abogados exitosos no se puede llegar a construir una imagen completa de las trayectorias de todos los abogados, pero este subgrupo ofrece la oportunidad de captar las de un grupo importante que, desde la perspectiva del éxito, sirve como referente para los abogados en su conjunto. Asimismo, mediante el análisis de los relatos sobre sus trayectorias laborales en 
instituciones de educación superior se puede también caracterizar la cultura académica de la disciplina en cuestión. Por estas razones resulta pertinente estudiar las trayectorias de abogados exitosos dedicados exclusivamente a la práctica profesional, así como las de aquellos dedicados a la academia dentro de las universidades, ya que ambas seguramente están relacionadas por tener un mismo campo disciplinario y profesional como referente común.

\section{Método}

Para dar respuesta a las preguntas de investigación se trabajó en una universidad de tamaño medio del noroeste de México, considerando la relativa facilidad de acceso a la institución para contactar a los entrevistados, ya que se contaba con el apoyo de algunos académicos y autoridades. Este escenario ofreció un contexto adecuado para el estudio y, al mismo tiempo, representó la oportunidad de generar información potencialmente útil para contribuir al conocimiento de los académicos del resto del país. La universidad en la que se trabajó cuenta con varios campus, más de 100 programas, una matrícula de más de 35.000 estudiantes, y una planta, incluyendo a profesores por horas, de alrededor de 4.000 académicos. La disciplina estudiada se imparte desde hace cuarenta años en la institución y actualmente cuenta con una matrícula de más de 3.000 estudiantes.

Para la realización del estudio se tomó como referente metodológico la investigación cualitativa, la cual usualmente toma muestras pequeñas de participantes para dar cuenta de microprocesos sociales que describen la realidad percibida por los individuos estudiados. Se empleó la Teoría Fundamentada (Strauss, 1987), que ofrece una estrategia para abordar estudios de cultura organizacional. Su propuesta se caracteriza por un análisis sistemático e intensivo, con frecuencias frase por frase y oración por oración, de entrevistas, notas de campo, observaciones y documentos pertinentes al objeto de estudio (Spradley, 1979; Sierra, 1998; Strauss y Corbin, 1998).

En el estudio más amplio se analizaron las carreras de Ingeniería, Biología, Lengua y Literatura y Derecho. Las carreras se eligieron a partir de la clasificación de disciplinas de Biglan (1973) y 
de los antecedentes históricos y tradiciones de las carreras dentro de la institución. De la combinación de ciencias básica-dura se seleccionó la carrera de Biología; de la básica-blanda la carrera de Literatura; de la aplicada-dura las de Ingeniería y de la combinación de aplicadablanda se seleccionó la carrera de Derecho. En el proceso de selección se consideró -y esto es algo que debe tenerse en cuenta al momento de apreciar los resultados y su universalidad-que los entrevistados fueran profesionales y académicos exitosos en sus respectivos medios.

Para cada carrera se seleccionaron seis participantes, de los cuales tres tendrían una dedicación completa a su práctica profesional y tres se dedicarían al trabajo académico de tiempo completo. En total fueron 24 profesionales. Los entrevistados que se dedicaban a la práctica profesional fueron seleccionados mediante la señalización por parte de otros profesionales que pertenecían a alguna agrupación reconocida en el Estado. De esta manera se identificaron como miembros distinguidos de la comunidad profesional correspondiente.

Para la selección de los tres entrevistados con énfasis en el trabajo académico se consideró el criterio de que fueran señalados por otros académicos como reconocidos por sus colegas. Más específicamente, se tomó en consideración que reunieran los requisitos de contar con tiempo completo en la institución y que (a) no ocuparan un puesto administrativo; (b) que tuvieran al menos cinco años de laborar en la institución como personal de tiempo completo, y (c) que fueran señalados como elementos distinguidos por su comunidad académica, lo cual se estableció realizando entrevistas exploratorias con diversos miembros de la misma. Para todos los casos se buscó, de la manera más equitativa posible, tomar en cuenta los aspectos de género, orientación hacia la investigación, docencia o práctica profesional y grado académico.

Una vez que se identificó a los participantes con énfasis en la práctica profesional y los participantes en la academia, se les contactó de manera personal y se les solicitó su colaboración para ser entrevistados. Las entrevistas tuvieron una duración promedio de 75 minutos cada una y se transcribieron textualmente conforme se fueron aplicando, con el fin de enriquecer las entrevistas posteriores 
258 TRAYECTORIAS DE ABOGADOS PROFESIONALES Y ACADÉMICOS - Esperanza Viloria, Jesús Galaz

con las observaciones que emergieran del análisis de las mismas. Para esto se utilizó el programa ATLAS Ti 5.0, un software diseñado para transcribir entrevistas y para servir de apoyo en el análisis cualitativo de las mismas.

En este estudio se utilizó una entrevista semiestructurada que abordó: (1) las características del trabajo que desarrolla el profesional; (2) los procesos de incorporación a la profesión o a la disciplina académica; (3) los criterios de estratificación dentro de la profesión académica y de la profesión; (4) la relación con diferentes actores de su contexto (colegas, estudiantes, personal dentro de la profesión y/o el trabajo académico administrativo, autoridades, institución como un todo, gremios, entre otros), y (5) el papel de la educación superior. De dichos aspectos emergieron las categorías abordadas en el presente informe.

\section{Resultados y discusión}

Antes de iniciar el análisis de los resultados debe enfatizarse que las categorías $^{3}$ reportadas se construyeron sobre la base de diferentes referentes contextuales, antecedentes y los informes proporcionados por los entrevistados, quienes las expresaron en función de sus interrelaciones, formación profesional y experiencia en la vida laboral. En este sentido, estas categorías se asocian con la trayectoria de los entrevistados de la carrera de Derecho.

Luego de presentar un breve perfil de los entrevistados se abordan, para cada tipo de profesional considerado, las trayectorias, sus diferentes periodos y los procesos identificados en ellos.

\section{Características de los entrevistados}

Los seis abogados entrevistados fueron cuatro hombres y dos mujeres, una en cada uno de los grupos de profesionales. Los tres entrevistados con dedicación exclusiva en la profesión, "abogados profesionales",

3 "Categoría" se refiere a los conceptos que representan un fenómeno. Dichos conceptos son las ideas centrales en los datos producto de las conceptualizaciones de acontecimientos, actos y sus efectos, manifestadas por los participantes (Strauss y Corbin, 1998). 
contaban con el grado de licenciatura y tenían en promedio 55 años y más de 35 años de experiencia profesional. En cambio, dos de los tres entrevistados que concentran su actividad en la academia, "abogados académicos", tenían posgrado. La edad promedio que tenían al momento de ser entrevistados era de 50 años y contaban con más de 30 años de experiencia profesional y académica. Este perfil no es típico de todos los abogados, y ello debe tenerse presente.

\section{Trayectorias profesionales}

Se describen en un primer momento las trayectorias de los abogados profesionales y, en un segundo, las trayectorias de los abogados académicos. Estas últimas se subdividen, en función del análisis de las entrevistas realizadas, en los abogados profesionales-académicos y los abogados académico-profesionales.

\section{Abogados profesionales}

Las trayectorias de los abogados profesionales se refieren a quienes se dedican de manera exclusiva al ejercicio profesional en el campo del Derecho. Sus trayectorias se organizaron en tres grandes periodos, los cuales se establecieron sobre base del análisis de entrevistas. El primer periodo es el de "decisión e ingreso a la carrera", que es cuando toman la decisión de estudiar la carrera e ingresan a ella. El segundo es el de "formación profesional", al que los entrevistados se refieren como "de estudios profesionales". El tercero es el de "egreso y actividad profesional", que se inicia cuando terminan sus estudios y comienzan su vida profesional. Este dura el resto de la vida laboral de estos abogados (ver tabla 1). 
260 TRAYECTORIAS DE ABOGADOS PROFESIONALES Y ACADÉMICOS - Esperanza Viloria, Jesús Galaz

Tabla 1. Trayectoria profesional de los abogados con dedicación a la práctica profesional (profesionales del Derecho) y los centrados en la actividad académica (abogados académicos).

\begin{tabular}{|c|c|c|c|c|c|}
\hline \multirow{2}{*}{$\begin{array}{l}\text { Tipo de dedicación a } \\
\text { la práctica profesional } \\
\text { Abogados } \\
\text { profesionales }\end{array}$} & \multicolumn{5}{|l|}{ Tiempo } \\
\hline & \begin{tabular}{|l|} 
1. Decisión e \\
ingreso
\end{tabular} & $\begin{array}{l}\text { 2. Formación } \\
\text { profesional }\end{array}$ & \multicolumn{3}{|c|}{ 3. Egreso y actividad profesional } \\
\hline \multirow{3}{*}{$\begin{array}{l}\text { Abogados } \\
\text { profesionales- } \\
\text { académicos }\end{array}$} & \multirow[t]{3}{*}{$\begin{array}{l}\text { 1. Decisión e } \\
\text { ingreso }\end{array}$} & \multirow[t]{3}{*}{$\begin{array}{l}\text { 2. Formación } \\
\text { profesional }\end{array}$} & \multirow{3}{*}{$\begin{array}{l}\text { 3. Egreso } \\
\text { y actividad } \\
\text { profesional }\end{array}$} & $\begin{array}{l}\text { 4. Ingreso a } \\
\text { la academia }\end{array}$ & $\begin{array}{l}\text { 5. Carrera } \\
\text { académica }\end{array}$ \\
\hline & & & & Práctica & Práctica \\
\hline & & & & Academia & Academia \\
\hline $\begin{array}{l}\text { Abogados académico- } \\
\text { profesionales }\end{array}$ & $\begin{array}{l}\text { 1. Decisión e } \\
\text { ingreso }\end{array}$ & $\begin{array}{l}\text { 2. Formación } \\
\text { profesional }\end{array}$ & \multicolumn{3}{|c|}{ 3. Egreso y actividad académica } \\
\hline
\end{tabular}

En el periodo de decisión e ingreso a la carrera los entrevistados reconocen una fuerte influencia de sus padres y, particularmente, del tipo de actividad laboral que éstos realizaban. El hecho de que el padre fuera abogado o contara con educación formal, como es el caso de los padres con preparación de maestro normalista, fue determinante para que los entrevistados continuaran estudios de abogacía. Se identificaron dos tipos de herencia familiar: "rica disciplinaria" y "rica no disciplinaria."

La influencia familiar jugó un papel importante no sólo para el ingreso a la carrera, sino también para la permanencia en ella, así como para la incorporación inmediata al campo laboral. Todo ello parece estar asociado, sobre todo, al proceso de socialización e identificación con la actividad práctica de la profesión y, particularmente, a la socialización respecto de los valores propios de la disciplina, los cuales son modelados por la herencia familiar "rica disciplinaria" asociada a la profesión del padre. Asimismo, también se trasmiten aspectos cognitivos, ya que se aprende el trabajo haciéndolo, generando niveles de confianza en la realización de la tarea apoyada y orientada por los padres. Por otro lado, la herencia familiar va más allá de los lazos familiares, ya que también se adquieren las amistades y vínculos con colegas de los padres que son heredados junto con una actividad profesional, cartera de clientes y el prestigio ganado durante un buen número de años en ella. Todo ello contribuye a que se materialice una trayectoria profesional "exitosa" del futuro abogado. 
Así, en el caso de uno de los entrevistados el padre era abogado y él decidió estudiar la carrera de Derecho por su influencia.

"Cuando estudié la carrera ya tenía conocimientos prácticos adquiridos en el bufete de mi papá. Conocía a varios de los maestros, ya que acudía a los juzgados o me relacionaba con ellos en las reuniones de trabajo con mi papá (...) la escuela fue un trámite para poder ejercer" (licenciado en Derecho 5:8).

La "herencia familiar no disciplinaria" se refiere a que los padres del entrevistado tenían una formación educativa alta, pero no asociada específicamente al campo del Derecho. La formación educativa en cuestión estuvo asociada a los valores de la mística de servicio a la comunidad, relacionada con la vocación de maestro de los padres de algunos de los abogados entrevistados. Así, para estos sujetos estudiar la carrera tuvo una fuerte motivación en la idea de servir a otros, pero desde una instancia pública, de un estatus o nivel de decisión que les ofrecía la formación en Derecho y el ejercicio de la profesión en puestos de gobierno.

Un efecto de una herencia familiar, tanto rica disciplinaria como rica no disciplinaria, es una ruta lineal de la trayectoria profesional, con periodos y etapas de desarrollo acelerado, ya que en ambos casos estos abogados se colocaron en el escenario laboral en corto tiempo. Estas condiciones del desarrollo de las trayectorias influyen mucho en la alta valoración de la especialización práctica atribuida a las habilidades personales, formación autónoma y competencia.

En el periodo de formación profesional se destaca el proceso de socialización recibido de los profesores con los que trabajaron en las diferentes instituciones, ya fueran públicas o privadas, durante el tiempo en que realizaron sus estudios profesionales. En dicho periodo recibieron la formación disciplinaria en la universidad y, sobre todo, tuvieron un contacto con la práctica desde el último año de la carrera, mediado por los profesores con los que trabajaron. Producto de la relación con los abogados profesores, hacia el final de la carrera se gestaron dos procesos; el de orientación y el de "padrinazgo" de los profesores de los entrevistados. Estos procesos contribuirían, 
262 TRAYECTORIAS DE ABOGADOS PROFESIONALES Y ACADÉMICOS - Esperanza Viloria, Jesús Galaz

posteriormente y de una manera positiva, a la incorporación casi inmediata al campo laboral al poco tiempo de egresar.

La orientación se presentó en el contexto de la tutoría recibida en el entrenamiento del trabajo disciplinario, al trabajar como ayudantes en despachos o en instancias como los juzgados. En estos espacios, los futuros abogados realizaban tareas menores relacionadas directamente con la profesión y, al mismo tiempo, aprendían las lógicas de los puestos, las rutas para el ingreso, las áreas de decisión y las habilidades sociales claves que debían desarrollar.

"Desde los primeros semestres busqué la oportunidad (...), el servicio social me la dio, logré conseguir trabajar primero con un secretario y más tarde con un magistrado, quienes fueron mis guías" (licenciado en Derecho 3:7).

Por otro lado, el padrinazgo ${ }^{4}$ se refiere al apoyo recibido por otro abogado o por alguna autoridad que, por lo general, detentaba un rango de poder o estatus en alguna institución. Este tipo de apoyo fue otorgado por el abogado "padrino" en la medida en que se construía una relación profesional a lo largo del trabajo de colaboración, cuando aún no egresaba el futuro abogado, y consistió en aportar alguna recomendación escrita o verbal para lograr obtener un puesto de trabajo. En algunos casos también convergieron varios elementos, entre los que destacan el interés personal, habilidades, convocatoria para el puesto y la recomendación del funcionario hecha a otra autoridad para que se considerara positivamente la incorporación de su "ahijado" a un puesto. De acuerdo con los entrevistados, la mayoría de las veces se les asignó un puesto menor en la escala de jerarquía de la institución pública.

"durante el último año de la carrera, uno de los abogados con los que trabajé me ayudó dándome una recomendación para trabajar en una institución pública, eso permitió que se abrieran algunas puertas" (licenciado en Derecho).

4 El padrinazgo es un concepto coloquial que se usa para referirse al apoyo, beneficios o protección recibida de una persona con poder o influencia. 
El periodo denominado egreso y actividad profesional se inicia cuando termina la carrera y los abogados comienzan su trabajo profesional. En esta etapa se capitalizan tres aspectos: la herencia familiar, ya sea del tipo rica disciplinaria o rica no disciplinaria, la formación profesional y la socialización de los entrevistados con las actividades profesionales, mediada por abogados dedicados al ejercicio de la profesión. Se capitaliza en la medida en que los factores en cuestión facilitan la rápida incorporación a la práctica profesional y, una vez en ella, la permanencia y avance en el empleo casi de manera definitiva y continua, que refleja altos niveles de especialización en el ámbito laboral, así como el desarrollo de habilidades relacionadas con la función desempeñada.

"he sido una persona muy activa socialmente, gusto de estrechar relaciones, lo que en este momento, que lo comento, me hace pensar que eso me permitió irme colocando. Por ejemplo, cuando egresé, un magistrado me dio una recomendación, que fue muy valiosa para mi contratación (...) en ese tiempo las cosas así se manejaban" (licenciado en Derecho 8:10).

\section{Abogados académicos}

Los abogados académicos son aquellos entrevistados cuya dedicación se concentra en la actividad universitaria. En función de las trayectorias manifestadas por los entrevistados, se ubicaron en dos tipos; los abogados profesionales-académicos y los abogados académicoprofesionales (ver tabla 1).

\section{Abogados profesionales-académicos}

La trayectoria de estos entrevistados se refiere a quienes realizan las dos actividades de manera simultánea, la práctica profesional y el trabajo académico, pero con mayor concentración en la actividad profesional. Dicha trayectoria se organizó en cinco periodos de desarrollo. El primero, al igual que el de los abogados profesionales, se inició con el proceso de la "decisión e ingreso a la carrera", que también en estos casos es influenciada por los padres, quienes eran maestros de primaria y secundaría. Según informan los entrevistados, ellos los impulsaron 
264 TRAYECTORIAS DE ABOGADOS PROFESIONALES Y ACADÉMICOS - Esperanza Viloria, Jesús Galaz

para estudiar una carrera profesional. La influencia familiar es tan importante que, en el caso de un abogado, sus padres eligieron para él Derecho, ya que para ellos era una carrera en la que podría tener un mejor trabajo y escalar económica y socialmente en una región que estaba en expansión.

"mi papá, un hombre muy culto que era maestro, me orientó y ayudó para inscribirme en la Escuela de Derecho. Decía que la carrera era una de las más reconocidas y necesarias (...) sobre todo (en esta región)" (licenciado en Derecho 9:2).

El segundo periodo, el de "formación profesional", representó para estos abogados una etapa de desarrollo centrada en lo individual y en el aprendizaje del campo de conocimiento elegido. Como en el caso de los abogados profesionales, la especialización en Derecho los puso en contacto con el trabajo de su modelo disciplinario, pero, a diferencia de ellos, estos entrevistados mencionan que entraron en contacto no solamente con la dimensión profesional de su carrera, sino también con las tareas del trabajo académico, así como con los valores profesionales de la enseñanza y la investigación. Este proceso de involucramiento de los estudiantes con estas tareas parece haber sido una manera efectiva de fomentar positivamente el interés de los futuros egresados para continuar con la carrera académica, como lo manifestaron los entrevistados y otros estudios sobre académicos (Galaz Fontes, 1999).

Los valores y conocimientos a los que estos abogados se expusieron fueron modelados por sus maestros y por maestrosfuncionarios con quienes trabajaron como ayudantes, ya fuera como auxiliares en sus clases en la universidad o como asociados o colaboradores en sus bufetes. Ello les permitió adquirir una experiencia profesional y académica valiosa. Como lo comentó un entrevistado:

"Durante casi dos años trabajé con maestros en la UNAM"; ayudaba con los cursos en clase, calificando trabajos, elaborando cursos o colaborando en sus despachos" (doctor en Derecho 8:4).

5 Universidad Nacional Autónoma de México. 
En esta segunda etapa el proceso de socialización tiene un fuerte componente personal, ya que ellos tomaron la iniciativa de buscar trabajo con maestros en la universidad. Los académicos con los que trabajaron eran personas de la vida política del país o abogados con reconocimiento nacional o internacional. Sin embargo, el trabajo con maestros destacados tuvo como principal interés los contactos fuera de la vida académica, sobre todo en los primeros años de su vida laboral. Dicho proceso de socialización en la academia y su motivación personal los colocó de manera natural en un contexto para realizar tanto actividades profesionales como docencia.

"desde que ingresé a la universidad, busqué y busqué ser asociado de un maestro que trabajó muchos años de magistrado (...), sabía que su experiencia y relaciones me ayudarían en mi carrera y no me equivoqué" (maestro en Derecho, 12:40).

"una de las cosas que valoro de mi formación en la UNAM fue tener la oportunidad de trabajar con personalidades de la vida pública y académica del país (...) aprendí del Derecho, de la política y de la docencia" (doctor en Derecho: 9: 12).

El tercer periodo para los abogados profesionales-académicos se denominó de "egreso y actividad profesional" y se caracterizó por una rápida incorporación al trabajo en despachos, en la judicatura o en puestos públicos, producto de la capitalización de las relaciones acumuladas durante actividades realizadas con funcionarios y académicos que participaban en la vida política. En este contexto, el tipo y nivel de actividad que desarrollaban los colocó, cierto número de años después, en un escenario ideal para estar en contacto con autoridades universitarias, que los consideraron como licenciados en Derecho con experiencia, por lo que fueron invitados a trabajar en la universidad. Tal momento marca el inicio del cuarto periodo de la trayectoria de los entrevistados.

Este cuarto periodo se considera como un momento crítico en el cual el abogado profesional-académico toma la decisión de trabajar a "tiempo completo" en la universidad y continuar con su actividad 
266 TRAYECTORIAS DE ABOGADOS PROFESIONALES Y ACADÉMICOS - Esperanza Viloria, Jesús Galaz

laboral, también de "tiempo completo", fuera de la universidad. Los aspectos que valoraron para aceptar la invitación fueron la libertad para organizar sus actividades, poder elegir el tipo de curso que impartir, el número de horas de dedicación y el horario, así como la flexibilidad para acudir a la escuela sin complicaciones laborales, la oportunidad de formar un grupo de especialistas en un área y el contacto con estudiantes. Esto último les ofrecía, asimismo, contar con recursos humanos para integrarlos a sus despachos o como ayudantes en las diferentes actividades que realizaban fuera de la universidad.

Los entrevistados comentaron que era bien visto que se desempeñaran como docentes y funcionarios en el gobierno. Según refieren, en la universidad pública es una costumbre que se mantiene hasta la fecha y es una práctica valorada por los académicos de la facultad. Los entrevistados señalan que dicha tradición también fue fomentada porque se le veía como una manera de promover la incorporación de los estudiantes a la práctica profesional. Entre los abogados, dichas prácticas son procesos culturales comunes y han sido reportadas por otros estudios como "lealtad" recíproca: los abogados, en la medida en que van ampliando su círculo en la universidad y en el trabajo, buscan promoverse hacia la burocracia o a otros puestos, y ayudan a quienes los ayudaron (Cleaves, 1985).

"desempeñé varios puestos en el gobierno municipal y
varios de mis estudiantes más destacados trabajaban en
mi oficina. La experiencia que adquirieron les permitió
conseguir un puesto de trabajo cuando egresaron"
(doctor en Derecho $4: 10$ ).

El quinto y último periodo se denomina "actividad académica", ya que los entrevistados concentran su actividad laboral en la docencia y corresponde al momento en que se ven enfrentados a diferentes decisiones, como la cercana jubilación o retiro de las actividades como funcionarios, por lo que deciden concentrar su actividad en las labores académicas. Esto es, los abogados, después de varios años de desarrollar simultáneamente el ejercicio profesional y el de tiempo completo en la universidad, lo hacen efectivo en este periodo. Concentrar su trabajo en la vida académica es un momento importante para los abogados, ya que deben tomar en cuenta la 
dinámica universitaria para el desarrollo de la siguiente etapa de su carrera dentro de la institución.

Los abogados profesionales-académicos del Derecho perciben tres formas de avanzar en su "nueva" carrera académica. La primera se asocia con las reglas de escalafón de los reglamentos, las cuales establecen la forma de ascenso en el contexto de la incorporación a las políticas educativas nacionales sobre investigación y evaluación de la productividad. Esta ruta es la menos importante para los entrevistados, ya que por la trayectoria mostrada hasta esos momentos, así como por el grado académico que se requiere para avanzar, no es la mejor opción, considerando su edad, área de experiencia y actividad.

La segunda es hacer carrera como funcionario en el ámbito administrativo (como director, subdirector o rector), lo cual depende, según su percepción, del capital político y motivación personal con que cuenta el académico para aspirar a dichos puestos. Como lo describe uno de los entrevistados:

"durante mi carrera dentro de la universidad he sido director de la facultad en dos ocasiones y también me he desempeñado como jefe en otras áreas de gestión de la misma universidad" (doctor en Derecho 12:22).

Una tercera ruta para avanzar es que los ahora académicos concentren su trabajo en la docencia y la vinculación asociada, y que se beneficien del prestigio capitalizado en ámbitos extrauniversitarios. No obstante, los entrevistados reconocen que las actividades implicadas en esta trayectoria no son muy reconocidas ni recompensadas.

"las rutas para escalar en la academia dependen de tus objetivos personales. Lo que sí es claro es que si te dedicas sólo a la docencia, el reconocimiento y el nivel en la jerarquía no es reconocido ni con el salario" (doctor en Derecho 9:4).

\section{Abogados académico-profesionales}

La trayectoria de los abogados académico-profesionales -quienes optan por dedicarse de manera exclusiva al trabajo como académicos 
268 TRAYECTORIAS DE ABOGADOS PROFESIONALES Y ACADÉMICOS - Esperanza Viloria, Jesús Galaz

en el sistema de educación superior poco después de haber egresadopuede describirse en tres periodos, los cuales parecen estar afectados por un fuerte factor asociado al género, como se observará en las entrevistas que dan sentido a esta subclasificación de los abogados académicos.

El primer periodo, llamado como en los otros casos "de decisión e ingreso", se ubica en el momento de elección de la carrera, la que se ve afectada por motivaciones personales asociadas con las condiciones familiares, caracterizadas por un menor capital social, ya que sus condiciones económicas y educativas eran considerablemente menos favorables que la de los profesionales del Derecho. En contraste con éstos, la herencia familiar para los abogados académicoprofesionales no fue rica y, en ese contexto, los contextos y procesos externos a la familia fueron un factor más importante en cuanto a su trayectoria profesional. Para el caso de una de las entrevistadas, la carrera de Derecho representó, además de la posibilidad de obtener conocimientos sobre las leyes y la aplicación de la justicia en la comunidad, la posibilidad de migrar a otro Estado y de escalar social y económicamente.

"desde que entré a la preparatoria pensé en estudiar la carrera de Derecho, ya que uno de los maestros que me dio clases me motivó. Sabía que no era fácil para una mujer dicho campo, pero representaba la oportunidad de salir de la comunidad en la que vivía y mejorar la vida familiar" (licenciada en Derecho 5:16).

El periodo "de formación profesional" es muy similar al descrito por los otros entrevistados, ya que también lo ubicaron durante su formación universitaria. Durante este tiempo el proceso de socialización y de orientación de sus profesores, junto con el reconocimiento de las habilidades personales de docencia, fueron los elementos claves para su rápida incorporación a la academia, primero en el sistema de educación media y, luego de varios años, en la academia universitaria.

A diferencia de todos los otros profesionales descritos, en el tercer periodo -el de egreso y actividad profesional- los abogados 
académico-profesionales se concentran en la carrera académica casi de inmediato al terminar los estudios universitarios. Contrariamente a los abogados profesionales-académicos, la elección para ingresar a la universidad se debió a su experiencia en la docencia, si bien es invitada por el director en turno, de manera similar al resto de los entrevistados. La invitación para laborar como maestra de tiempo completo fue hecha al poco tiempo de haber egresado. Como se ha mencionado, las razones son diferentes a las de los abogados profesionales-académicos, ya que en este caso se reconoce su experiencia como docente en educación media y, sobre todo, los conocimientos que tenía sobre temas educativos.

La decisión de aceptar el trabajo de académico de tiempo completo se asocia con la motivación personal de trabajar como docente y participar en las actividades de apoyo como tutora, en el diseño del plan de estudios en su área disciplinaria o en la administración educativa y, asimismo, continuar su formación en un posgrado en el área de educación.

Las anteriores condiciones se observan en el caso de una entrevistada que ingresó a la universidad hace más de veinte años. Esta abogada, desde su inicio como académica, ha distribuido su tiempo de dedicación entre la docencia, las actividades de apoyo (como la elaboración de manuales, cartas descriptivas y análisis de planes de estudio) y coordinar algunas otras actividades académicas. Sus tiempos y tipos de dedicación han variado, pero regularmente el porcentaje a la docencia es el mayoritario y el más constante.

Una entrevistada reconoce en su trayectoria profesional vías similares a los abogados profesionales-académicos. No obstante, señala algunas diferencias que existen en las vías para el desarrollo de la carrera académica dentro de la universidad. Como en el caso de los abogados profesionales-académicos, está claro para ella que una forma de escalar en la jerarquía académica es ajustándose a la normatividad universitaria, la cual estipula los requisitos que debe cumplir para, por ejemplo, pasar de una categoría de Asociado C a otra de Titular A. Otra forma para avanzar en la carrera la asocia con las actividades de gestión, y una más a la vida política fuera 
270 TRAYECTORIAS DE ABOGADOS PROFESIONALES Y ACADÉMICOS - Esperanza Viloria, Jesús Galaz

y dentro de la universidad, siendo esta última la más valorada en sus colegas. Respecto de la primera vía de avance, la entrevistada comenta que tiene muy claro que para poder avanzar en la carrera académica debe:

"tener doctorado, publicar, tutoría y formar recursos humanos en investigación, como lo marcan los estatutos, pero en mi caso, no ha sido la ruta seguida. Varios maestros, como en mi caso, se han concentrado en la docencia y en las actividades de apoyo, actividades poco valoradas por colegas y autoridades universitarias (...) quizá en la beca (PREDEPA) se reconoce parte del esfuerzo del trabajo, pero falta mucho" (maestra en Derecho).

Sí bien reconoce la relevancia que pueden tener la gestión y la vida política como vías para avanzar en la profesión académica, considera que su trayectoria y prestigio los ha conseguido por la vía de la docencia. También enfatiza que existe una tendencia de género hacia el trabajo docente, ya que las abogadas con las que trabaja se han especializado en temas relacionados con educación, enseñanza del Derecho o didáctica de la profesión. Sin embargo, considera que es poco valorada la dedicación exclusiva a la docencia por parte del gremio de abogados.

"las licenciadas que nos hemos dedicado a la docencia somos poco valoradas por los abogados, (quienes) consideran que nos hace falta el ejercicio en la práctica de manera permanente como ellos. Estoy convencida de que mi labor es importante, sobre todo cuando hablamos de la enseñanza de los valores en el aprendizaje del Derecho" (licenciada en Derecho, 31:9).

En su caso, la universidad representó la posibilidad de estar en contacto con los conocimientos de la disciplina y con profesionales destacados. También reconoce que le ha dado la posibilidad de combinar su trabajo con ser esposa y madre. La flexibilidad de horarios y tareas que demanda su trabajo le ha sido compatible con sus actividades personales, situación que es compartida por otras mujeres en el campo de la medicina (Lester, 2008). Como la entrevista lo expone: 
"mire licenciada, siendo realistas la universidad, verdad, me da la oportunidad de organizar mi vida personal y realizar un trabajo. Para las mujeres abogadas, sobre todo cuando tenemos hijos, contar con un horario fijo, vacaciones, años sabáticos, son buenas condiciones de trabajo. Sin embargo, creo que el salario es poco" (licenciada en Derecho, 24:39).

\section{Conclusiones}

Las categorías presentadas muestran una variedad de dimensiones sobre las trayectorias de los abogados profesionales y académicos que, de manera sistemática, implicaron algunos procesos consistentes, en general, con otros estudios realizados en otras disciplinas, como serían los procesos de socialización a la profesión, la influencia de la familia y el escalar socialmente (García Salord, Landesmann y Gil Antón, 1993; Gil Antón et al., 1994).

La consistencia de dichos resultados resulta interesante, puesto que la presente investigación parte de entrevistas individuales a un número pequeño de abogados exitosos en las que se exploraron sus trayectorias profesionales en diferentes contextos organizacionales y sociales. Las entrevistas permitieron también identificar algunos valores y supuestos de los abogados que les dan identidad, lo cual no obstó para documentar una diversidad en sus trayectorias profesionales. Es importante tener presente, sin embargo, que tales trayectorias han sido identificadas en abogados que, además de ser considerados exitosos, tienen una edad promedio de 55 años y una experiencia en su campo de trabajo de más de 35 años.

Las trayectorias de los abogados muestran una diversidad considerable en sus características y dimensiones. En el caso de los entrevistados, sin embargo, se puede señalar que las trayectorias mostraron una vía casi lineal que se puede apreciar en las rutas descritas, en particular en el caso de la incorporación inmediata al trabajo al momento de egresar. Así, el avance es -desde una perspectiva temporal amplia-continuo y ordenado, y está integrado por diferentes periodos y niveles de desarrollo profesional. Conviene recordar de nuevo que este estudio consideró a profesionales exitosos, lo que 
272 TRAYECTORIAS DE ABOGADOS PROFESIONALES Y ACADÉMICOS - Esperanza Viloria, Jesús Galaz

puede explicar las trayectorias continuas, ordenadas y lineales.

Para la determinación de los periodos descritos se consideraron los puntos críticos comunes referidos por los entrevistados. Así, el primero se ubica en la decisión e ingreso a la carrera; el segundo en la formación profesional en la que toma decisiones de tipo disciplinarios y de relaciones sociales por construir, ambas asociadas a la vida profesional; el tercero se refiere al de egreso e incorporación a la actividad profesional, donde deben elegir el área laboral que mejor responda a sus intereses personales en cuanto a habilidades, estatus, remuneración económica y/o poder. Dichos puntos críticos se ven matizados por la herencia familiar, por los niveles de socialización personal y por el tipo de orientación o padrinazgo recibido.

A diferencia de lo que sucede con los abogados profesionales, en las trayectorias de los abogados académicos se destacan dos tipos de trayectoria: la de los profesionales- académicos y la de los académicosprofesionales. Los abogados profesionales-académicos se distinguen por realizar, luego de un buen número de años de estar dedicados de manera prácticamente exclusiva a la práctica profesional, dos actividades de manera simultánea, como son el ejercicio profesional y el trabajo como académico de tiempo completo, durante cierto número de años. Los entrevistados reportan que transitan por varias etapas, en las cuales las trayectorias dentro de la universidad tienen una movilidad horizontal, ya que han desempeñado varias actividades (docencia, gestión, vinculación) en un mismo nivel de la estructura organizacional. Ello ha hecho que su avance en la jerarquía académica sea más lento y prolongado, sobre todo cuando se habla de las actividades de docencia e investigación, ya que en el caso de gestión han ocupado puestos directivos en diferentes periodos y niveles de la jerarquía institucional, siendo el avance en este rubro más rápido y permanente.

El segundo tipo de trayectoria reportado por los abogados académicos implica una dedicación de manera prácticamente exclusiva al trabajo en el sistema de educación superior. Los entrevistados conciben en señalar que esta trayectoria se desarrolla en tres periodos. El primero, de decisión e ingreso a la carrera, es similar a lo reportado por todos los abogados entrevistados, pero se puede destacar que las condiciones 
familiares y habilidades personales fueron diferentes, ya que algunos de ellos contaban con el referente académico de los padres que eran maestros normalistas. En el segundo periodo, de formación profesional, en el cual sucede la socialización a la profesión y posteriormente una rápida incorporación al mercado laboral. Se puede destacar que los abogados profesionales no tuvieron una socialización significativa en la vida académica; en cambio los académico-profesionales estuvieron en contacto con las actividades de docencia y, en algunos casos, con la gestión. La importancia asignada al periodo de formación profesional por los dedicados a la academia habla, entonces, de la necesidad de estudiar esta etapa como una en la que se puede promover la carrera académica mediante políticas para el fomento y selección temprana de profesionales (Galaz Fontes, 1999).

Una segunda diferencia crítica entre los abogados profesionalesacadémicos y los académicos-profesionales se ubica en el tercer periodo, en el egreso y actividad profesional, en el cual el ejercicio profesional para los segundos es muy breve y, más bien, la carrera laboral se concentra en la vida académica, a la que se incorporan de manera similar que el resto de los abogados que lo hacen, ya que fueron invitados por su experiencia y reconocimiento profesional. Sin embargo, concentran su actividad en la docencia y en las actividades de apoyo, las cuales valoran de manera importante, más que continuar por el escalafón universitario o la vida política. En particular, el trabajo de las mujeres en dicha actividad es poco reconocido por sus pares y tienen pocas oportunidades de desarrollo laboral (Lester, 2008).

En términos generales, la anterior descripción indica que las trayectorias se encuentran asociadas a los aspectos culturales, tanto entre los abogados profesionales como entre los abogados académicos, ya que muestran algunas semejanzas y diferencias relacionadas con el contexto organizacional en el que se desarrollan.

Entre los aspectos culturales similares se pueden destacar los procesos de socialización y relaciones interpersonales para el desarrollo de la profesión. Los factores culturales en que difieren se relacionan con los mecanismos de selección y avance en la carrera, ya que ambos procesos dependen de la institución en que realizan su actividad 
274 TRAYECTORIAS DE ABOGADOS PROFESIONALES Y ACADÉMICOS - Esperanza Viloria, Jesús Galaz

profesional. Se puede destacar que el entorno es factor que interviene en la trayectoria de los profesionales y de los académicos; sin embargo, un elemento que influye de manera importante en las trayectorias de los entrevistados que concentran su actividad en la academia son sus motivaciones personales de desarrollo profesional.

En este sentido, resulta relevante continuar explorando el desarrollo de la carrera profesional y sus procesos para abundar en el conocimiento de la cultura académica y ayudar a comprender el complejo espacio de relaciones de significados entre los diversos sujetos sociales (Ibarra Colado, 2001). En la medida en que se identifiquen las creencias, valores y mecanismos utilizados por los abogados exitosos se pueden generar escenarios para el acceso a las organizaciones, a grupos de pares y avanzar en la carrera dentro de las universidades. También se pueden establecer los mecanismos más sutiles para la selección de los futuros académicos dentro de las instituciones educativas.

La complejidad y diversidad descrita respecto de diferentes trayectorias profesionales en el contexto de las organizaciones ofrece un escenario para continuar con estudios cualitativos que identifiquen los diferentes procesos culturales de los campos profesionales y las culturas académicas. En particular, es necesario detallar más aún las formas de incorporación y avance, tanto en la profesión como en la academia, con la seguridad de que este tipo de conocimiento coadyuvará al conocimiento y desarrollo de la profesión académica y de la educación superior en general.

\section{Referencias bibliográficas}

Atlas.Ti. V5.0. (2004) The Knowledge Workbench. Alemania; Scientific Software Development.

Austin, E.A. (1989) Faculty Cultures. En Clark, B.R. (Ed.) The encyclopedia of higher education: An Encyclopedia (Vol. 3, pp. 1614-1622). New York: Pergamon Press.

Becher, T. \& Trowler, P.R. (2001) Academic tribes and territories (2 $\left.{ }^{\text {nd }} e d.\right)$ Great Britain: The Society for Research into Higher Education and Open University Press. 
Biglan, A. (1973) Relationships between subject matter characteristics and the structure and output of university departments. Journal of Applied Psychology, 57(3), pp. 204-213.

Buenrostro Ceballos, A. (1991) Los pasos ganados; ensayos y testimonios para la historia de la Universidad Autónoma de Baja California. México: Universidad Autónoma de Baja California.

Chavoya Peña, M.L. (2001) Organización del trabajo y culturas académicas. Estudio de dos grupos de investigadores de la universidad de Guadalajara. Revista Mexicana de Investigación Educativa, 6(11), pp. 79-93.

Clark, B. R. (1987) The academic life: Small worlds, different worlds. Princeton, NJ: The Carnegie Foundation for the Advancement of Teaching.

Clark, B. R. (2004) Sustaining Change in Universities: Continuities in case studies and concepts. United States of America: The Society for Research into Higher Education; Open University Press.

Cleaves, P. (1985) Las profesiones y el Estado: el caso de México. México: El Colegio de México.

Galaz Fontes, J. F. (1999) University experience and academic profession. Revista Electrónica de Investigación Educativa, 1(1). Sitio en Internet. Disponible en http://redie.uabc.mx/vollnol/contents-galaz.html

Galaz Fontes, J. F. (2003) La satisfacción laboral de los académicos en una universidad estatal pública; la realidad institucional bajo la lente del profesor. México: Asociación Nacional de Universidades e Instituciones de Educación Superior.

Galaz Fontes, J. F. y Viloria Hernández, E. (2004) La toma de decisiones en una universidad pública estatal desde la perspectiva de sus académicos. Revista Mexicana de Investigación Educativa, IX (22), pp. 637-663.

García Salord, S.; Landesmann, M. y Gil Antón, M. (1993) Académicos. México: Segundo Congreso Nacional de Investigación Educativa.

García Salord, S.; Grediaga Kuri, R y Landesmann Segall, M. (2003) Los académicos en México. Hacia la construcción de un campo de conocimiento. En Ducoing Watty P. (Coord.). Sujetos, Actores y procesos de Formación. México: Consejo Mexicano de Investigación Educativa.

García Salord, S. (2001) Las trayectorias académicas: de la diversidad a la heterogeneidad. Revista Mexicana de Investigación Educativa, Vol. 6(11), pp. 15-31. 
276 TRAYECTORIAS DE ABOGADOS PROFESIONALES Y ACADÉMICOS - Esperanza Viloria, Jesús Galaz

Garritz Ruiz, A. (1997) Reflexiones sobre dos perfiles universitarios: El docente y el investigador. Revista de la Educación Superior, XXVI (102), pp. 9-25.

Gil Antón, M., et al. (1994) Los rasgos de la diversidad: Un estudio sobre los académicos mexicanos. México: Universidad Autónoma Metropolitana, Unidad Azcapotzalco.

Gil Antón, M. (2000) Los académicos en los noventa: ¿actores, sujetos, espectadores o rehenes? Revista Electrónica de Investigación Educativa, 2(1). Sitio en Internet. Disponible en http/redie.ens.uabc.mx/vol2nol/ contenido-gil. Html

Grediaga, Kuri, R. (2001) Retos y condiciones de desarrollo: la profesión académica en México en la última década. Revista Mexicana de Investigación Educativa, 6(11), pp. 95-117.

Grediaga, Kuri, R. (2001) Condiciones y perspectivas de desarrollo de la profesión académica en México. Revista de la Educación Superior, XXX (117), pp. 23-48.

Ibarra Colado, E. (2001) La universidad en México hoy: gubernamentalidad y modernización. México: Universidad Nacional Autónoma de México; Universidad Autónoma Metropolitana, Unidad Iztapalapa; Asociación Nacional de Universidades e Instituciones de Educación Superior.

Lester, J. (2008) Performing gender in the workplace. Community College Review, 35(4), pp. 277-305.

Landesmann S.M. (2001) Trayectorias académicas generacionales: constitución y diversificación del oficio académico. Revista Mexicana de Investigación Educativa, 6(11), pp. 33-61.

Light, D.W.; Marsden L.R. y Corl T.C. (1990) A Framework for academic Careers. En Finkelstein (Ed.) Faculty and faculty issues in colleges and universities. Needham Heights, Massachusetts: Ginn Press.

Padilla González, L. E. (2007) La socialización del personal académico. Fortaleciendo la profesión académica en México. Revista de la Educación Superior, XXXVI (142) 142, pp. 87-100.

Piñera Ramírez, D. (1997) Historia de la Universidad Autónoma de Baja California 1957-1997. México: Universidad Autónoma de Baja California

Robbins, S. (2004) Comportamiento organizacional. México: Pearson.

Schein, E.H. (2004/1989) Organizacional cultura and leadership. United States of America: Jossey-Bass.

Sierra, F. (1998). Función y sentido de la entrevista cualitativa en investigación 
social. En Galindo Cáceres J. (Coord.) Técnicas de investigación en sociedad, cultura y comunicación. México: Pearson, Addison Wesley Longman, pp. 277-345.

Spradley, J.P. (1979) The ethnographic interview. United States of America: Holt, Rinehart and Winston.

Strauss, A.L. (1987) Qualitative analysis for social scientists. United States of America: New York: Cambridge University Press.

Strauss, A. y Corbin, J. (1998) Basics of qualitative research. Techniques and procedures for developing grounded theory. United States of America: Sage Publications.

Tapia Uribe, M. (2000) Equilibrio y consenso en el gobierno de la universidad pública en México. En Cazés Menache, D.; Ibarra Colado, E. y Porter Galetar L. (Coords.) Evaluación, financiamiento y gobierno de la universidad: El papel de las politicas. México: Universidad Nacional Autónoma de México, Centro de Investigaciones Interdisciplinarias en Ciencias y Humanidades, pp. 113-124.

Tierney, W.G. (1993) Building Communities of difference: Higher education in the twenty-first century. United States of America; The Johns Hopkins University Press.

Vargas Leyva, R. (2000) Trayectorias profesionales de los ingenieros en la industria maquiladora electrónica: el caso de Sanyo Video Componentes. Revista Electrónica de Investigación Educativa, 2(2). Sitio en Internet. Disponible en http://redie.uabc.mx./vol2no2/contenidovargas.html

Villa Lever, L. (2001) El mercado académico: La incorporación, la definitividad y las promociones, pasos para una misma trayectoria de formación. Revista Mexicana de Investigación Educativa, 6(11), pp. 63-77.

Recibido: 4 de mayo de 2009

Aceptado: 26 de mayo de 2009 